\title{
USE OF HARMONICS IN RF CAVITIES IN MUON CAPTURE FOR A NEUTRINO FACTORY OR MUON COLLIDER *
}

\author{
D. Neuffer, C. Yoshikawa, Fermilab, Batavia, IL 60510, USA \\ R. Johnson, Muons, Inc., Batavia IL 60510, USA
}

\begin{abstract}
Common to various front end designs for a muon collider or neutrino factory are costly low frequency RF cavities used to bunch muons. In this paper we show that adding higher harmonic RF cavities to the bunching section of a muon capture channel can provide as good or better bunching efficiency than the case where only the fundamental is used. Since higher harmonic cavities are less expensive to build and operate, this approach implies significant cost savings.
\end{abstract}

\section{INTRODUCTION}

For a neutrino factory or muon collider, short, intense bunches of protons are focused onto a target to produce pions, which decay into muons that are then accelerated into a high-energy storage ring. In a muon collider, muons and anti-muons are collided; in a neutrino factory, the muons decay to provide beams of high-energy neutrinos $[1,2,3]$. In either case, the challenge is to collect and accelerate as many muons as possible. The pions (and resulting muons) are initially produced within a short bunch length but with broad transverse and longitudinal energy spreads, much larger than the acceptance of any accelerator. In this paper we consider improving the ability to capture the longitudinal energy spread with higher frequency cavities. Since the aperture of such cavities is reduced, this approach assumes that their transverse acceptance is adequate for a sufficient subset of muons or that transverse beam cooling is involved.

In the portion of the front end addressed by this strategy, pions and muons drift from the production target, lengthening into a long bunch with a high-energy "head" and a low-energy "tail". These particles would then be placed in an accelerating system that decelerates the head and accelerates the tail and forms the beam into a bunch that can then be cooled and accelerated for the $\mu^{+}-\mu^{-}$Collider or neutrino factory.

\section{APPARATUS AND CHALLENGE}

The front end system up to and including the low frequency RF (LFRF) portion that is under study here is shown in Figure 1. Eight $\mathrm{GeV}$ protons are targeted onto a $30 \mathrm{~cm}$ long Fe rod that is encapsulated in a $20 \mathrm{~T}$ solenoid. Pions created from the target (and a very small number of prompt muons) are captured by having their transverse component of momentum rotated into the longitudinal as they traverse the $10 \mathrm{~m}$ long tapered solenoid which starts at $20 \mathrm{~T}$ and $7.5 \mathrm{~cm}$ radius at the target and tapers off to 2
$\mathrm{T}$ and $22.5 \mathrm{~cm}$ radius at the far end, where the RF cavities begin. The LFRF cavity section stretches for up to 100 meters, with each cavity being $20 \mathrm{~cm}$ long, operating with maximum gradient of $3 \mathrm{MV} / \mathrm{m}$, and for purposes of this study, is a simple pillbox. The baseline RF frequency is $25 \mathrm{MHz}$. The entire LFRF cavity region is surrounded by a $2 \mathrm{~T}$ solenoid to contain the charged particles. Simulations were performed in G4beamline [4].

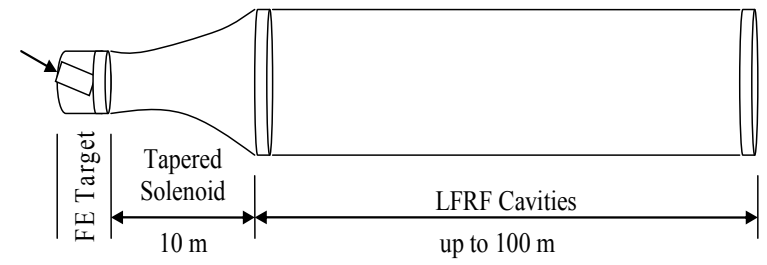

Figure 1: Simulated apparatus for Study of Harmonics in LFRF Cavities. The $8 \mathrm{GeV}$ proton beam and $30 \mathrm{~cm}$ long Fe target are aligned together, but are $100 \mathrm{mrad}$ off angle with respect to the axis of the tapered solenoid $(10 \mathrm{~m}$ long) and LFRF cavities.

Muons and pions exiting the tapered solenoid exhibit the long bunch structure shown in Figure 2, consisting of a high-energy head for early arriving particles and a low energy tail for late arrivals. The challenge is to reduce the muon and pion momentum spread to maximize the yield of muons in the overall process.

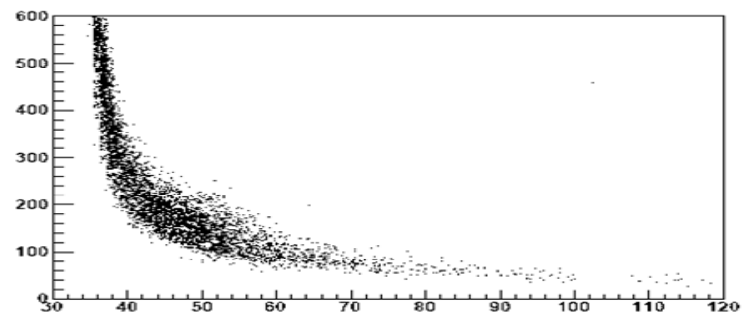

Figure 2: Momentum $[\mathrm{MeV} / \mathrm{c}]$ on vertical axis vs. time of arrival [nsec] on horizontal axis for $\mu^{+} \mathrm{s}+\pi^{+} \mathrm{s}$ at end of the tapered solenoid.

\section{STRATEGY FOR LOW RF CAVITIES}

The basic concept is straightforward and has been previously studied [5], although not from the present perspective of cutting costs. The idea is to widen the effective portion of the E field over which bunching of muons and pions take place. A "sawtooth" RF waveform, as shown in Figure 3, should be optimal. 


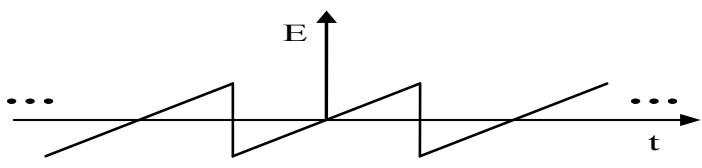

Figure 3: Idealized sawtooth waveform that the LFRF cavities will approximate.

Since the RF cavities operate at a single frequency, our strategy is to create a lattice structure that incorporates prorated amounts of RF cavities at particular harmonics following the Fourier transform of the sawtooth waveform. The form of the Fourier transform is:

$$
F(\text { sawtooth }) \approx \sum_{n=1}^{\infty}(-1) \quad(1 / n) \sin \left(n f_{0} t\right)
$$

G4beamline is used to study the effect of adding the second harmonic (up to $n=2$ in equation 1 ) by introducing an RF cavity operating at double the base frequency every second, third, fourth, or fifth cell. Based on equation 1, incorporating an RF cell operating at double the base frequency should occur once every third cell. In the present model each RF cell has equal length $(20 \mathrm{~cm})$ and gradient $(3 \mathrm{MV} / \mathrm{m})$, but differing harmonic frequencies. Effects of adding third and fourth harmonic cells are also evaluated. Construction of the RF lattice to incorporate up to the third harmonic will be illustrated; the same method applies to create lattices with higher harmonics. Table 1 shows how the weight associated with each harmonic is derived as well as the corresponding size of the lattice, which is $11 \mathrm{RF}$ cells for this case. Figure 4 displays the layout of a lattice following such weights. The algorithm places cells with same harmonic at uniform spacing, with lower $\mathrm{n}$ value prevailing due to its higher weight where there is a conflict in cavity placement. Figure 5 shows the resultant yield of $\mu^{+}+\pi^{+}$for cases of free drift (no RF implemented), single harmonic (25 $\mathrm{MHz}$ ), incorporating second harmonic at varying intervals, and implementations including third and fourth harmonics of $25 \mathrm{MHz}$. Table 2 lists the maximum yield for each of the cases. A resultant RF lattice to implement in a muon collider or neutrino factory would extend to an optimal length of 20 to 30 meters.

Table 1: Weights of frequencies in constructing RF lattices incorporating up to third harmonic. Second column is weight associated with particular frequency as given by Fourier Transform in equation 1. Third column is weight normalized by sum of weights of the 3 frequencies involved. Denominator in third column, 11, is size of lattice

\begin{tabular}{|l|l|l||}
\hline \hline Harmonic $n$ & Weight $\sim 1 / \mathrm{n}$ & $\begin{array}{c}\text { Weight with respect to top } 3 \\
\text { frequencies }\end{array}$ \\
\hline \hline 1 & $1 / 1=6 / 6$ & $(6 / 6) /(6 / 6+3 / 6+2 / 6)=6 / 11$ \\
\hline 2 & $1 / 2=3 / 6$ & $(3 / 6) /(6 / 6+3 / 6+2 / 6)=3 / 11$ \\
\hline 3 & $1 / 3=2 / 6$ & $(2 / 6) /(6 / 6+3 / 6+2 / 6)=2 / 11$ \\
\hline
\end{tabular}

\begin{tabular}{|l|l|l|l|l|l|l|l|l|l|l|l|}
\hline Cell in Lattice & 1 & 2 & 3 & 4 & 5 & 6 & 7 & 8 & 9 & 10 & 11 \\
\hline Harmonic $\mathrm{n}$ & 1 & 2 & 1 & 3 & 1 & 2 & 1 & 3 & 1 & 2 & 1 \\
\hline
\end{tabular}

Figure 4: Layout of RF cells in a lattice with up to third harmonic.

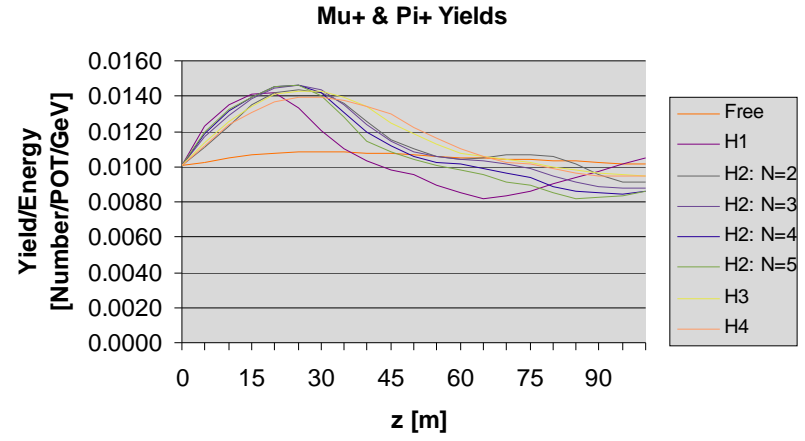

Figure 5: Yield of $\mu^{+}+\pi^{+}$with $100 \mathrm{MeV} / \mathrm{c} \leq$ momentum $\leq 200 \mathrm{MeV} / \mathrm{c}$. "Free" refers to no RF applied; $\mu^{+} \mathrm{s}$ and $\pi^{+} \mathrm{s}$ drift freely. "H1" corresponds to case of only base frequency of $25 \mathrm{MHz}$. "H2: $\mathrm{N}=\mathrm{n}$ " refers to case of second harmonic cavity being placed every nth cell. "H3" refers to use of up to third harmonic as illustrated in Figure 4. "H4" refers to use of up to fourth harmonic, which has a lattice of size 25 cells. Statistical error is approximately $2 \%$ of the yield values.

Table 2: Maximum yields of lattices utilizing various harmonic schemes.

\begin{tabular}{|l|l|l|}
\hline \hline Lattice Type & $\begin{array}{c}\text { Max Yield of } \mu^{+} \& \pi^{+} / \\
\text {Proton on Target } / \mathrm{GeV}\end{array}$ & Optimal Depth (meters) \\
\hline \hline Free Drift & $1.09+/-0.02 \%$ & $30-35$ \\
\hline $\mathrm{H} 1$ & $1.42+/-0.03 \%$ & 20 \\
\hline $\mathrm{H} 2: \mathrm{N}=2$ & $1.44+/-0.03 \%$ & 25 \\
\hline $\mathrm{H} 2: \mathrm{N}=3$ & $1.46+/-0.03 \%$ & 25 \\
\hline $\mathrm{H} 2: \mathrm{N}=4$ & $1.46+/-0.03 \%$ & 25 \\
\hline $\mathrm{H} 2: \mathrm{N}=5$ & $1.46+/-0.03 \%$ & $20-25$ \\
\hline $\mathrm{H} 3$ & $1.43+/-0.03 \%$ & 25 \\
\hline $\mathrm{H} 4$ & $1.39+/-0.03 \%$ & $25-30$ \\
\hline
\end{tabular}

Although the various RF scenarios provide similar yields numerically, there are qualitative properties that may reveal quantitative differences downstream when further stages are involved. Figure 6 shows momentum versus time for select cases. Optimal yields for all cases incorporating multi-harmonics occurred $\sim 25 \mathrm{~m}$ from the start of the RF section. At that distance, Figure 6(a) shows that the spread in time has increased for freely drifting $\mu^{+} s+\pi^{+} s$ when compared to these particles first entering the RF cavities in Figure 1. Figure 7 illustrates the analytical E field associated with the lattices for cases displayed in Figure 6. For example, Figure 6(b) shows p vs. $t$ for application of RF with base harmonic; Figure 7(b) displays the simple corresponding sine wave. The time period of the base $25 \mathrm{MHz}$ frequency, and hence all other cases implementing harmonics, is 40 nsec. In case of $\mathrm{H1}$, the effective portion of the sinusoid that has the correct slope direction to coalesce $\mu^{+} s+\pi^{+} s$ is only half of the $40 \mathrm{nsec}$ period. Figure 6(b) reveals a coalescing 
window that is consistent with $\sim 20$ nsec. Figures 6 (c) and 7(c) show a consistent picture of coalescing where an opposite sign for slope in middle of coalescing window results in a thinning of the momentum band at the center of the window. Results in Figures 6(d,e,f) show a flatter distribution in momentum, which is desired and can be explained by their correspondingly better $\mathrm{E}$ field approximations in Figures $7(\mathrm{~d}, \mathrm{e}, \mathrm{f})$ to the sawtooth waveform in Figure 3. Qualitatively, it is expected that a momentum spread that is more uniform will result in better overall capture efficiency when other elements downstream are incorporated.
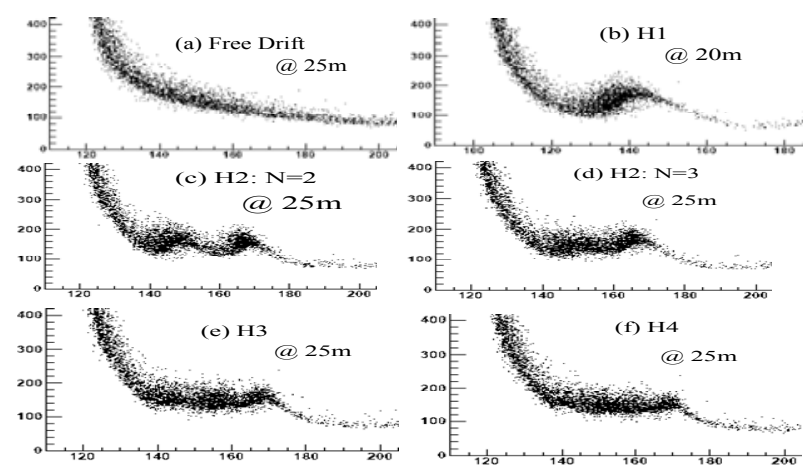

Figure 6: Momentum (MeV/c) versus time (nsec) for $\mu^{+} \mathrm{s}$ $+\pi^{+}$s. (a) Freely drifting $\mu^{+} s+\pi^{+} s$ at optimum $z=25$ meters for cases incorporating harmonics. (b) RF with $\mathrm{H} 1$ at optimal yield, $z=20$ meters. (c)-(f) Select harmonic configurations at $z=25$ meters. The caption for Figure 5 contains the explanation of $\mathrm{H}$ and $\mathrm{N}$ conventions.
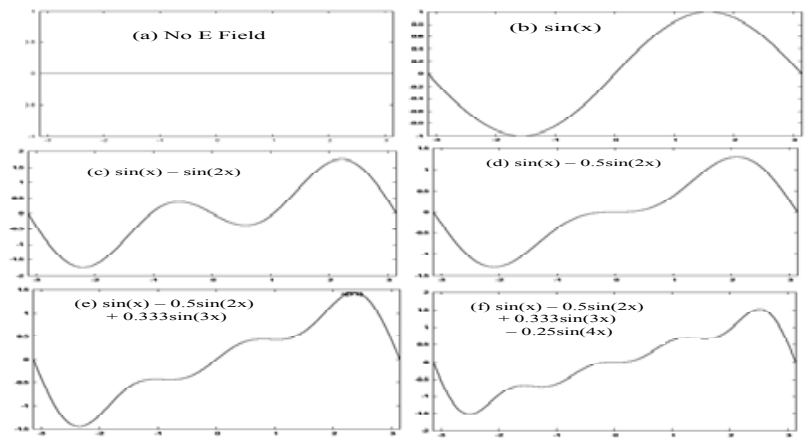

Figure 7: Analytic expressions of $\mathrm{E}$ field in various harmonic configurations. Cases correspond to those in Figure 6. Range on the $\mathrm{x}$ axis is a $25 \mathrm{MHz}$ RF cycle (40ns).

\section{COST CONSIDERATIONS}

A critical criterion for RF configurations is cost. Assuming cost of construction and operation (wall plug power) scales with the volume of the RF elements (or stored energy) and that the cavity radius is inversely proportional to the frequency, one can calculate relative costs for these multi-harmonic lattices. Table 3 contains results of such calculation. Although the $\mathrm{H} 2 \mathrm{~N}=4,5$ lattices are cheaper per unit length than $\mathrm{H} 1$, they cannot make up for cost of added length of $5 \mathrm{~m}$. However, other multi-harmonic lattices are more cost effective than $\mathrm{H} 1$. Note that the size of the lattice for H3 is 11 RF cells, H4 is 25 , and $\mathrm{H} 5$ is 137 . Because of relatively small advantages and this greater complexity, we do not consider using 5 or more harmonics.

Table 3: Yields and relative costs of harmonic lattices. Cost analysis only assumes cost scales with cavity volume.

\begin{tabular}{||l||l|l|}
\hline \hline \multicolumn{1}{|c||}{ Lattice Type } & $\begin{array}{c}\text { Max Yield of } \mu^{+} \& \pi^{+} / \\
\text {Proton on Target } / \mathrm{GeV}\end{array}$ & $\begin{array}{c}\text { RF Cost Relative to } \\
\text { Using Single Frequency }\end{array}$ \\
\hline \hline Free Drift & $1.09+/-0.02 \%$ & $0 \%$ \\
\hline $\mathrm{H} 1$ & $1.42+/-0.03 \%$ & $100 \%$ \\
\hline $\mathrm{H} 2: \mathrm{N}=2$ & $1.44+/-0.03 \%$ & $78.13 \%$ \\
\hline $\mathrm{H} 2: \mathrm{N}=3$ & $1.46+/-0.03 \%$ & $93.75 \%$ \\
\hline $\mathrm{H} 2: \mathrm{N}=4$ & $1.46+/-0.03 \%$ & $101.56 \%$ \\
\hline $\mathrm{H} 2: \mathrm{N}=5$ & $1.46+/-0.03 \%$ & $106.25 \%$ \\
\hline $\mathrm{H} 3$ & $1.43+/-0.03 \%$ & $79.23 \%$ \\
\hline $\mathrm{H} 4$ & $1.39+/-0.03 \%$ & $70.66 \%$ \\
\hline
\end{tabular}

\section{CONCLUSION}

As demonstrated above, incorporation of RF with base harmonic or multi-harmonics considered in various ways yielded similar results. However, distributions in $\mathrm{p}$ vs. $\mathrm{t}$ show qualitative differences that reveal potentially higher yields with the use of higher harmonics. Introducing cost into the decision reveals an even larger impetus to use higher harmonics. The lattice for H4 (25) provides 29\% reduction in cost compared to a lattice using only the base $\mathrm{RF}$, and this lattice has the potential for higher yields downstream. Hence, if the front end design for a future muon collider or neutrino factory uses LFRF, an H4 type lattice (as in Figure 8) could be used to significantly lower costs and provide at least as good or better efficiency.

\begin{tabular}{|l|c|c|c|c|c|c|c|c|c|c|}
\hline Cell in Lattice & 1 & 2 & 3 & 4 & 5 & 6 & 7 & 8 & 9 & 10 \\
\hline Harmonic $n$ & 1 & 4 & 1 & 2 & 1 & 3 & 1 & 2 & 1 & 3 \\
\hline Cell in Lattice & 11 & 12 & 13 & 14 & 15 & 16 & 17 & 18 & 19 & 20 \\
\hline Harmonic $\mathrm{n}$ & 1 & 2 & 1 & 4 & 1 & 2 & 1 & 3 & 1 & 2 \\
\hline
\end{tabular}
\begin{tabular}{|l|c|c|c|c|c|c|}
\hline Cell in Lattice & 21 & 22 & 23 & 24 & 25 \\
\hline Harmonic $\mathrm{n}$ & 1 & 4 & 1 & 2 & 3 \\
\hline
\end{tabular}

Figure 8: Layout of RF cells in the lattice to incorporate frequencies up to the fourth harmonic.

The study here applied equal E field gradients for each harmonic. In general, we expect to be able to obtain higher gradients at higher frequencies. Future investigations will reveal if this can be exploited to use higher gradients in a smaller number of higher frequency RF cavities such that the overall weight assigned to the harmonic is maintained, resulting in further cost reduction.

\section{REFERENCES}

[1] "Cost-effective Design for a Neutrino Factory", with J. S. Berg et al., Phys. Rev. STAB 9,011001(2006)

[2] N. Holtkamp and D. Finley, eds., A Feasibility Study of a Neutrino Source based on a Muon Storage Ring, Fermilab-Pub-00/108-E (2000). 
[3] S. Ozaki, R. Palmer, M. S. Zisman, eds., BNL-52623, June 2001. http://www.cap.bnl.gov/mumu/studyII/FS2report.html

[4] G4beamline, http://g4beamline.muonsinc.com

[5] K. Paul, D. Neuffer, C. Ankenbrandt, unpublished paper, http://muonsinc.com 